\section{MS27-01 Structure refinement using precession electron diffraction tomography and dynamical diffraction theory \\ Lukas Palatinus}

1. Institute of Physics of the CAS, Na Slovance 2, 18221 Prague, Czechia

email:palat@fzu.cz

Electron diffraction techniques have made an enormous progress over the last decade. Currently it is possible to solve simple to medium sized crystal structures almost routinely using electron diffraction tomography techniques, and many examples of solving quite complex structures are also available. The bottle neck in the structure analysis work flow from electron diffraction was the optimization and validation of the structure model by structure refinement.

Recently, a possibility to perform full least-squares refinement of crystal structures against electron diffraction tomography data was studied in a series of works $[1,2,3]$. It was shown that it is indeed possible to obtain reliable structure models with good figures of merit from electron diffraction tomography data. The best results are obtained, if the data are recorded as a tilt series of diffraction patterns (electron diffraction tomography, EDT) combined with the precession of electron beam. On a series of test cases it was shown that the structure models deviate from the reference X-ray or neutron refinements on average by $0.02 \AA[3]$.

The method of structure refinement against electron diffraction tomography data (dynamical refinement for short), has been implemented in the crystallographic computing system Jana2006 [4] and made freely available to the scientific public. In the last months, considerable experience is being gained with this technique in several groups and on various materials. The results are encouraging. It has been shown that using this method it is possible to perform detailed crystallographic analyses of crystal structures, including the detection of partially occupied position, the refinement of site occupancies, determination of absolute structure and the use of difference Fourier synthesis to detect fine details of the structures, including the positions of hydrogen atoms. First applications of the technique have already been published [5,6] and more are in preparation.

[1] L. Palatinus et al., Acta Cryst. A69 (2013) 171.

[2] L. Palatinus et al., Acta Cryst. A71 (2015) 235.

[3] L. Palatinus et al., Acta Cryst B71 (2015) 740.

[4] V. Petricek et al., Z. Kristallgr. 229 (2014) 345.

[5] C. A. Correa et al., J. Alloys Comp. 672 (2016) 505. 